\title{
Intuiciones en lógica: una propuesta moderada
}

\author{
Intuitions in logic: a moderate proposal
}

\author{
Diego Tajer \\ IIF-SADAF-CONICET \\ diegotajer@gmail.com
}

\begin{abstract}
Resumen
Las intuiciones juegan un rol significativo en los debates sobre lógica. En este artículo, analizo cuán legítima es esa práctica. En la primera parte, distingo entre intuiciones teóricas y preteóricas, y argumento que algunas intuiciones preteóricas no deberían ser tomadas en cuenta para la lógica. En particular, nuestras intuiciones preteóricas sobre el concepto de validez no son particularmente relevantes, porque no tenemos un concepto uniforme o claro de validez en el lenguaje natural que pueda ser elucidado. Sin embargo, argumento que, dado que los conectivos son usados de forma homogénea en el discurso ordinario, podemos apelar a intuiciones preteóricas para establecer el significado de ellos en una teoría lógica. En la segunda parte del artículo, considero y doy respuesta a algunas objeciones. Dos de ellas intentan mostrar que, si la posición es adoptada, las intuiciones preteóricas sobre los conectivos terminan siendo poco confiables e inútiles. Una de ellas dice que esta posición mixta es inestable: las intuiciones preteóricas sobre los conectivos también son intuiciones preteóricas sobre la validez. El último problema está relacionado con la definición de validez y la posibilidad de revisarlo.
\end{abstract}

Palabras clave: intuiciones, consecuencia lógica, epistemología de la lógica, constantes lógicas.

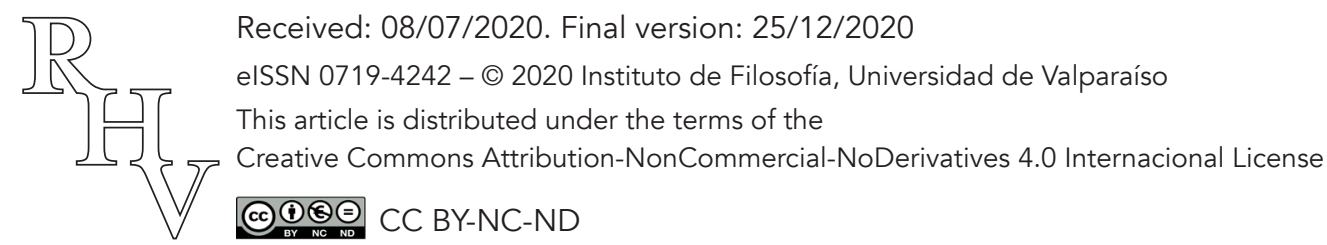


Intuiciones en lógica: una propuesta moderada

$$
\text { Diego Tajer }
$$

\begin{abstract}
Intuitions play a significant role in debates about logic. In this paper, I analyze how legitimate is that practice. In the first part of the paper, I distinguish between theoretical and pretheoretical intuitions, and argue that some pretheoretical intuitions are not to be taken into account in logic. Particularly, our pretheoretical intuitions about the concept of validity are not of much importance, since we don't have a uniform or clear concept of validity in the natural language to be elucidated. Nevertheless, I argue that, since logical connectives are more homogeneously used in our ordinary speech, we can appeal to pretheoretical intuitions to establish their meaning in a logical theory. In the second part of the paper, I consider and reply to four objections to this moderate proposal. Two of them try to show that, if this position is adopted, then the pretheoretical intuitions about the connectives are completely unreliable and useless. One of them argues that this mixed position is unstable: pretheoretical intuitions about the connectives are also pretheoretical intuitions about validity. The last problem is related to the definition of validity and the possibility of revising it.
\end{abstract}

Keywords: intuitions, logical consequence, epistemology of logic, logical constants.

\title{
1. Las intuiciones y su rol en la filosofía
}

Las intuiciones juegan un rol importante en la metodología de la filosofía analítica. Frecuentemente son utilizadas como piedra de toque para evaluar teorías o propuestas filosóficas. Pero la fundamentación de esta práctica no es muy clara. El rol epistémico de las intuiciones suele ponerse en cuestión. Ni un físico, ni un biólogo, ni un sociólogo, dirían que sus disciplinas usan las intuiciones de los científicos (o de la gente común) como evidencia; por el contrario, una y otra vez intentan refutar las intuiciones que tienen. Y esto no es visto como problemático.

¿Por qué, entonces, la filosofía tiene derecho a usar las intuiciones para justificar sus afirmaciones? El debate ha generado distintas posiciones. En un extremo, están los que (Bealer 1998) consideran que las intuiciones filosóficas están conectadas a priori con la verdad, y que esto transforma a la intuición en un mecanismo confiable de adquisición de creencias. En otro extremo, filósofos como Cummins (1998) consideran que las intuiciones deben ser eliminadas de la práctica filosófica. Para Cummins, nuestras intuiciones filosóficas pueden ser tan erróneas como nuestras intuiciones físicas o biológicas. Posiciones similares son sostenidas por Weinberg, Nichols y Stich (2001), cuyos experimentos en psicología social mostraron que la intuición puede estar afectada por efectos de framing y trasfondos culturales. Entre medio de estas posiciones, filósofos como Sosa (1998), Williamson (2007) y Chudnoff (2011) consideran que la intuición, así como la percepción, solo provee justificación prima facie.

Revista de Humanidades de Valparaíso, 2020, No 16, 239-253

(c) (1) $\Theta$ CC BY-NC-ND 
Intuiciones en lógica: una propuesta moderada

Diego Tajer

Un aspecto importante del debate es qué entendemos en cada caso por "intuición". Para autores racionalistas como Bealer, existen "intuiciones racionales" mediante las cuales captamos el significado de ciertos conceptos, a partir de una facultad mental específica. Autores como Williamson toman a la intuición como un juicio o una disposición a juzgar $(2007,3)$. En psicología, es más usual entender una intuición como un juicio espontáneo y no-inferencial (Mercier \& Sperber 2011, 153). Para los propósitos de este artículo, no es esencial decidirse por una de estas opciones. Sin embargo, tomaré la menos comprometida metafísicamente, que es la de Williamson: una intuición es simplemente un juicio o una disposición a juzgar.

Una posición mucho más provocativa es adoptada por Cappelen (2012), quien sostiene que los filósofos no usamos predominantemente intuiciones, sino expresiones como "intuitivamente", que significan cosas muy distintas según el contexto. Cappelen afirma que el uso de estas expresiones es nocivo, porque si las tomamos demasiado literalmente nos terminan comprometiendo con una misteriosa facultad racional de "intuir". Según Cappelen, hablar de intuiciones "es un mal hábito y tenemos que abandonarlo" (2012, 50). En este artículo también entendemos que el uso de "intuición" en lógica es bastante impreciso, pero tomamos la teoría sencilla de Williamson donde una intuición es un juicio o inclinación a juzgar, por lo cual la posible variabilidad del significado de "intuición" en lógica no debería causar demasiados problemas.

Las intuiciones aparecen frecuentemente en debates sobre lógica filosófica. Quizás el caso más famoso sea el de Kripke, en Naming and Necessity (1980), donde la noción de intuición se utiliza numerosas veces. La teoría central, según Kripke, está basada en intuiciones: por ejemplo, la intuición de que "Aristóteles podría haber sido jardinero" pero "Aristóteles no podría no haber sido Aristóteles". Según Kripke, "tenemos una intuición directa de la rigidez de los nombres, exhibida en nuestra comprensión de las condiciones de verdad de esas oraciones" (1980, 22; la traducción y las cursivas son mías).

Cuando desarrollamos teorías de la verdad, intentamos definir el concepto de verdad más intuitivo posible. Por ejemplo, Horsten dice: "En general, es más fácil argumentar a favor de la debilidad veritativo-teorética de una teoría que a favor de su fuerza; uno puede señalar los principios veritativos que intuitivamente deberían ser demostrables pero que esa teoría no puede probar" (Horsten 2011, 26; la traducción y las cursivas son mías).

En discusiones sobre lógicas no-clásicas, los defensores de los enfoques no-clásicos suelen decir que son teorías "más intuitivas" que la lógica clásica. Por ejemplo, Mares dice: "La lógica relevante fue desarrollada en parte para evitar las paradojas de la implicación material y estricta. Estas fórmulas son teoremas de la lógica clásica, pero son anti-intuitivas" (Mares 2004, 8; la traducción y las cursivas son mías].

Finalmente, cuando discutimos sobre paradojas, intentamos proponer soluciones que sean lo más intuitivas posibles. Field afirma sobre las paradojas:

Revista de Humanidades de Valparaíso, 2020, No 16, 239-253

(c) $(1)(9)$ CC BY-NC-ND 
Intuiciones en lógica: una propuesta moderada

Diego Tajer

Lo que nos interesa es averiguar cómo podríamos modificar estos razonamientos: encontrar una nueva forma de razonamiento que nos resulte intuitivamente aceptable, pero que evite las conclusiones claramente inaceptables (Field 2009, 17; la traducción y las cursivas son mías).

¿Pero cuán legítimo es apelar a intuiciones en este contexto? En este artículo, voy a proponer que, con respecto a ciertos conceptos lógicos fundamentales, la utilidad de las intuiciones es muy limitada. Sin embargo, afirmaré que hay otros conceptos lógicos cuya investigación sí puede estar guiada por intuiciones.

\section{El rol de las intuiciones en lógica}

Mi principal oponente en esta discusión es Extremo, cuya tesis es la siguiente:

(Extremo) La lógica debe respetar todas nuestras intuiciones preteóricas sobre conceptos lógicos.

Extremo no representa necesariamente la posición de un lógico o filósofo en particular, sino simplemente una opción posible en el debate. Sin embargo, filósofos como Bealer (1998) y Goodman (1965) sostuvieron tesis similares.

Las intuiciones preteóricas son intuiciones que no surgen de la investigación teórica. Con este concepto intento distinguir entre Extremo y alguien que piensa que la lógica debería estar basada en intuiciones teóricas. No consideraría a esa última posición inadecuada $^{1}$, sino débil. Esto se debe a que las intuiciones teóricas de los lógicos pueden ser confundidas por los principios generales de la disciplina. Cuando uno apela (por ejemplo) a la intuición de que una prueba lógica debería ser finita, no hay ningún concepto serio de intuición en juego; más bien, se trata de un desiderátum usual en la disciplina.

Por ejemplo, uno podría decir que, en matemática pura, la intuición de los matemáticos juega un rol legítimo. Esto parece razonable. Y, de hecho, los matemáticos apelan a intuiciones teóricas, pero no hay intuiciones preteóricas sobre funciones o límites. Un matemático podría construir una teoría a partir de su intuición de que la Hipótesis del Continuo es falsa, o de que debería haber un conjunto universal; sin embargo, es difícil sostener que esas intuiciones existían antes de que aprendiera teoría de conjuntos, en algún tipo de trasfondo preteórico.

Una disciplina donde las intuiciones preteóricas son fundamentales, y donde la tesis de Extremo es al menos plausible, es la epistemología analítica. Los epistemólogos suelen apelar a intuiciones sobre conceptos ordinarios como "conocimiento", que pueden

\footnotetext{
${ }^{1}$ De hecho, dar un argumento contra esta posición sería muy difícil. El concepto de intuición teórica es muy vago, y podría extenderse para incluir cualquier intuición que fue adquirida con experimentos científicos o descubrimientos matemáticos.
}

Revista de Humanidades de Valparaíso, 2020, No 16, 239-253

(c) (1) @९ $\mathrm{O}$ CC BY-NC-ND 
Intuiciones en lógica: una propuesta moderada

Diego Tajer

ser contrastadas a las intuiciones de las personas sin formación filosófica ${ }^{2}$. Por ejemplo, (prácticamente) nadie afirma que uno puede conocer algo sin que eso sea verdadero. Por esa razón, esas intuiciones pueden funcionar como justificación.

En la misma línea, la filosofía experimental puede cuestionar esta práctica: un experimento que muestre que, para la mayoría de la gente, realmente hay conocimiento en el caso Gettier (un experimento mental que intenta mostrar un caso de creencia verdadera justificada sin conocimiento), podría preocupar a los epistemólogos analíticos ${ }^{3}$. La pregunta entonces es: respecto a las intuiciones preteóricas, ¿es la lógica como la epistemología analítica o como la matemática?

En lógica, donde la apelación a las intuiciones es ubicua, la diferencia entre intuiciones teóricas y preteóricas es más clara. Por ejemplo, uno puede defender la intuitividad (teórica) de la regla-omega (Tarski 1936), o la intuitividad del hecho de que no hay distintos puntos fijos Kripkeanos (Kripke 1975), pero esas son intuiciones teóricas. No es la misma intuición a la que apelamos cuando (por ejemplo) argumentamos a favor de la designación rígida a partir del hecho de que Sócrates no pudo haber sido distinto a Sócrates.

Esto no significa que en lógica nunca apelamos a intuiciones preteóricas. Por el contrario, lo hacemos usualmente. Algunos ejemplos son: la defensa del esquema-T ("La oración 'La nieve es blanca' es verdadera si y sólo si la nieve es blanca"); las paradojas de la implicación ("No es correcto afirmar que "Si tomo veneno, entonces si no tomo veneno, soy un dinosaurio"'), la tesis de que "el rey de Francia es católico" es indeterminada y no falsa; la premisa de Tolerancia en la paradoja sorites ("si le saco un granito de arena a un montón de arena, sigue siendo un montón de arena"), etc. En estos casos, contrastamos teorías filosóficas contra intuiciones preteóricas, que perfectamente podríamos tener incluso si no supiéramos filosofía. Lo deseable sería que nuestras teorías respeten esas intuiciones. En casos donde uno apela a intuiciones preteóricas, el peso de las intuiciones suele ser mucho mayor.

Lo que está realmente en disputa aquí es el concepto de Validez. Algunos filósofos sostienen que existe un concepto preteórico de validez, que es elucidado por nuestra teoría lógica. Típicamente, Tarski (1936) sostuvo esa visión, aunque existe una discusión exegética sobre cuán preteórica era la noción "ordinaria" de la que hablaba Tarski (cf. Jané 2005). El pluralismo lógico de Beall y Restall (2006) está basado en la idea de que hay una noción tarskiana preteórica de validez que se puede precisificar de distintas formas.

\footnotetext{
${ }^{2}$ Si bien este diagnóstico es compartido por la mayoría de los autores, hay algunas excepciones. En particular, Cappelen (2012) considera que el uso de intuiciones en filosofía analítica en general está sobreestimado; según su teoría, los filósofos no apelan a intuiciones generalmente, sino a argumentos.

${ }^{3}$ Este debate no está aún saldado. Algunos autores como Williamson (2007) consideran que la filosofía experimental no afecta a la filosofía porque las intuiciones de no-filósofos no deberían ser tomadas en cuenta con la misma importancia que las intuiciones de filósofos.
}

Revista de Humanidades de Valparaíso, 2020, No 16, 239-253

(c) $(1)(9)$ CC BY-NC-ND 
Intuiciones en lógica: una propuesta moderada

$$
\text { Diego Tajer }
$$

Aquí voy a defender que la lógica no tiene por qué respetar nuestras intuiciones preteóricas. Voy a rechazar (Extremo) porque, como voy a argumentar, el concepto general de Validez tiene un carácter teórico inescapable ${ }^{4}$. Esto no significa que no exista un concepto preteórico de validez; simplemente implica que puede existir, pero no es aquello que los lógicos intentan capturar. En otras palabras, no importa demasiado cuáles son nuestras intuiciones preteóricas sobre la validez.

La principal razón es la siguiente. Para tener valor justificatorio, las intuiciones preteóricas deben hablar sobre un concepto ordinario. Esto es obvio: nuestras intuiciones preteóricas sobre la gravedad o los cardinales infinitos, si es que las tenemos, no sirven de mucho. Pero no cualquier concepto ordinario es igual. Si queremos usar las intuiciones preteóricas como evidencia, lo primero que necesitamos es un concepto claro y uniforme del lenguaje natural, sobre el cual exista un mínimo de acuerdo (aunque sea implícito) entre hablantes competentes 5 . Por ejemplo, siguiendo las observaciones wittgensteinianas, no serviría usar nuestras intuiciones en un análisis de un concepto ramificado como juego, porque (presumiblemente) no hay propiedades en común entre los objetos que caen bajo esta categoría. De hecho, Weatherson (2003) sostiene que las intuiciones sobre el conocimiento, la propiedad, la justicia o la causalidad pueden usarse legítimamente como evidencia porque no son conceptos ramificados.

Pero desafortunadamente, la validez es un concepto ramificado como juego. Como muestran Mercier y Sperber (2011), nuestro concepto intuitivo de un argumento correcto consiste en una mezcla heterogénea entre criterios para evaluar inferencias siguiendo un fin dialógico. Desacordamos en la evaluación de argumentos simples. Esto se debe a que estamos preocupados en persuadir a los otros, no en realizar inferencias correctas desde un punto de vista teórico. Evans (2005) también considera que el razonamiento ordinario está orientado pragmáticamente, y esto explica por qué fallamos en realizar inferencias lógicas abstractas en muchas ocasiones; en otras palabras, "la gente común tiene una comprensión pobre de la necesidad lógica” (Evans 2005, 170-171).

La evaluación intuitiva de argumentos se basa en criterios muy distintos entre sí, que pueden incluir aspectos contextuales, fuerza retórica y conveniencia. Evaluamos argumentos constantemente, y tenemos intuiciones sobre la corrección de argumentos; pero nos falta una noción preteórica de validez que sea suficientemente clara y uniforme para

\footnotetext{
${ }^{4}$ Catarina Dutilh-Novaes (2011) argumenta a favor de una posición similar, aunque en defensa de una idea general distinta. Argumenta que deberíamos tomar a la historia y la filosofía de la lógica como fuentes para los criterios de validez.

${ }^{5}$ Williamson $(2007,191)$ argumenta que las intuiciones confusas de la gente no-experta no son realmente importantes, porque la filosofía debería apelar a las intuiciones de los expertos (i.e. filósofos), sobre las cuales hay más homogeneidad.
}

Revista de Humanidades de Valparaíso, 2020, No 16, 239-253

(c) $(1)(9)$ CC BY-NC-ND 
Intuiciones en lógica: una propuesta moderada

Diego Tajer

contar como objeto de un análisis conceptual. De acuerdo a la naturaleza misma del "concepto ordinario de validez", cualquier definición precisa del concepto de validez va a contar como incorrecta en distintos sentidos.

Lo que pasa con la validez no pasa con conceptos como la negación, la conjunción o la disyunción; estos son conceptos ordinarios que pueden ser usados en forma más homogénea. Por ende, es esperable que podamos encontrar definiciones de los conectivos que respeten nuestras intuiciones preteóricas. Por esta razón considero que, cuando hacemos lógica, las intuiciones preteóricas sobre el significado de los conectivos lógicos en lenguajes naturales pueden y deben ser tenidas en cuenta, y que ellas pueden ayudar a determinar cómo debería ser la lógica.

Mi propuesta puede ser formulada del siguiente modo:

(Moderado) La lógica debe respetar nuestras intuiciones preteóricas sobre los conectivos lógicos, pero no necesita respetar nuestras intuiciones sobre la validez.

Mi posición es compatible con la metodología de la mayoría de las discusiones en lógica. Algunos autores apelan a las intuiciones preteóricas sobre el uso de los condicionales para afirmar que el análisis clásico de los condicionales está mal. Por ejemplo, la oración "si tú vienes a la fiesta, entonces Julio César tenía ojos azules o no los tenía" no es intuitivamente verdadera (porque el hecho de que vengas a la fiesta no es relevante para la fisonomía de Julio César). Eso podría usarse a favor de un condicional relevantista. Lo mismo puede decirse sobre las consideraciones de McGee (1985) sobre el condicional: sus argumentos apelan a intuiciones preteóricas que supuestamente muestran premisas verdaderas y conclusión falsa en una instancia de Modus Ponens. Si el argumento fuera correcto, podría llevarnos a revisar el condicional.

Sin embargo, mi defensa del uso de intuiciones en estos casos no es radical. Respetar estas intuiciones no implica que no puedan ser discutidas: siempre debería ser posible reflexionar sobre ellas y postular un significado distinto. Las consideraciones metafísicas, por ejemplo, podrían servir para la revisión. Esto fue particularmente claro en la discusión sobre la negación. Algunos filósofos consideran que las oraciones sobre un futuro incierto no pueden ser verdaderas ni falsas. Entonces, si "en un millón de años habrá una guerra o no la habrá" no es todavía verdadera ni falsa, esto nos podría llevar a adoptar una negación distinta donde el tercero excluido no es un teorema. La revisión también puede partir de paradojas. Por ejemplo, los dialeteístas (Priest 2006) consideran que algunas oraciones paradójicas son verdaderas y falsas a la vez. Por eso, usan un concepto de negación que admite contradicciones. En esos casos, no sólo las evidencias preteóricas aparecen como evidencia, sino también otras consideraciones filosóficas. En otras palabras, las intuiciones sobre los conectivos lógicos deben ser respetadas, pero eso no significa que sean el único elemento a tener en cuenta.

Revista de Humanidades de Valparaíso, 2020, No 16, 239-253

(c) (1) @९ $\mathrm{O}$ CC BY-NC-ND 
Intuiciones en lógica: una propuesta moderada

Diego Tajer

Antes de pasar a las objeciones, haré dos observaciones relativas a discusiones filosóficas recientes. En primer lugar, frente a las objeciones de la filosofía experimental, que mostró que las intuiciones de los hablantes comunes no siempre van en línea con las de los filósofos, muchos autores (cf. Williamson 2007, 191) afirmaron que en filosofía lo importante son las intuiciones de los expertos, es decir, los filósofos profesionales. La idea de Williamson es interesante para aplicar a mi posición. Respecto a conceptos preteóricos como los conectivos lógicos, la intuición de los expertos posiblemente sea más confiable, pero la intuición de los que no conocen lógica también puede ser útil para desarrollar o contrastar teorías. Vale aclarar que un experto también puede tener intuiciones preteóricas sobre conceptos del lenguaje ordinario, aunque estarán seguramente afectadas por su conocimiento de la teoría. Como indica Williamson $(2007,191)$, esa “contaminación" es inevitable. Sin embargo, respecto a los conceptos teóricos como "validez", será la intuición del experto la que cuente principalmente, y esta "intuición" no será muy distinta de un principio general de una teoría. Naturalmente, las intuiciones de los expertos no tienen por qué converger hacia una teoría común; por eso, el valor de una "intuición teórica" también es relativo, en tanto los expertos tengan distintas intuiciones incompatibles entre sí. Eso nos lleva al próximo punto.

Recientemente, un asunto muy popular en filosofía de la lógica es la discusión sobre anti-excepcionalismo. Según el anti-excepcionalismo, la lógica no es especial respecto a otras ciencias: no es analítica ni a priori, es revisable y su dinámica es similar a la de una teoría científica. Según Martin (2020), esta posición se lleva mal con un enfoque fuerte sobre las intuiciones, como el racionalista. Si la principal evidencia de la lógica es la intuición a priori racional, entonces se trata de una disciplina excepcional. Es importante aclarar que nuestra visión aquí sobre las intuiciones es mucho más moderada, porque las tomamos como juicios o disposiciones a juzgar. De este modo, decir que las intuiciones funcionan como justificación en una determinada discusión (por ejemplo, en la discusión sobre el significado de los conectivos) no nos compromete con ningún tipo de excepcionalismo sobre la lógica. De hecho, la situación será similar a la de la lingüística. En lingüística, es común apelar a intuiciones para resolver cuestiones de gramaticalidad: por ejemplo, podemos afirmar que "Michi es unas azul" es una oración no-gramatical porque nos suena mal, o porque le suena mal al hablante competente promedio. Lo que no podemos es apelar a intuiciones respecto a conceptos teóricos: por ejemplo, "las lenguas romances declinan sus adjetivos en género y número" es una afirmación sobre la cual la intuición que uno tenga (especialmente, la intuición preteórica) carece de importancia. En ese sentido, la lógica no es excepcional, porque se comporta como otras disciplinas científicas.

En la próxima sección, voy a desarrollar y responder algunas posibles objeciones a mi posición. 
Intuiciones en lógica: una propuesta moderada

$$
\text { Diego Tajer }
$$

\section{Objeciones a la propuesta: validez, verdad y significado}

\section{1. ¿Sirven realmente las intuiciones para determinar el significado de los conectivos?}

Podría objetarse a mi posición que, para determinar el significado de los conectivos lógicos, la apelación a intuiciones también podría ser eliminada. Esta posición es extrema, contraria a la que defendí aquí. Si admitimos que las consideraciones metafísicas y teóricas pueden ser útiles para determinar el significado de los conectivos en una teoría lógica, ¿por qué seguimos apelando a intuiciones preteóricas, cuando pueden ser falsas?

A esto puedo responder que admitir factores adicionales para analizar el significado de los conectivos en la teoría no implica rechazar las intuiciones preteóricas como evidencia. A veces no necesitamos ninguna consideración externa. Por ejemplo, cuando queremos establecer si la paradoja positiva $p \rightarrow(q \rightarrow p)$ es una paradoja, podemos apelar solamente a nuestras intuiciones preteóricas sobre el uso del condicional: no suena razonable decir que "si va a llover mañana, entonces si en este momento desaparece el universo, va a llover mañana". Pero incluso en casos donde tomamos en cuenta las consideraciones preteóricas, es compatible hacer ambas cosas: nuestras intuiciones sobre el uso de esos conceptos, y los aspectos filosóficos que quizás ignoramos cuando no pensamos detenidamente en el asunto, pueden ser útiles para configurar el significado de los conectivos en nuestra teoría.

Supongamos que se rechaza el tercero excluido porque algunas oraciones sobre el futuro no son ni verdaderas ni falsas. Entonces, si nuestras intuiciones preteóricas sobre la disyunción y la negación fueran clásicas, no serían concluyentes para determinar el significado de esos conectivos en la teoría lógica (porque nos comprometerían con el tercero excluido, que ya rechazamos por razones metafísicas). Pero esto no implica dejar de respetar las intuiciones preteóricas sobre los conectivos. Porque necesitamos entender el significado de la oración para darnos cuenta por qué el futuro no determina su valor de verdad, y nuestra comprensión intuitiva de las expresiones lógicas en el lenguaje natural es esencial para hacerlo. Las consideraciones teóricas complementan las preteóricas, pero no las reemplazan.

Lo mismo sucede cuando nos enfrentamos con una paradoja semántica. Típicamente, tratamos de resolverla del modo más intuitivo posible, que es el más compatible con nuestras intuiciones preteóricas sobre los conceptos en el lenguaje natural (sea conectivos o predicados como Verdad).

De hecho, algunas lógicas no-clásicas surgen de intuiciones preteóricas. Especialmente, respecto a la negación. En determinados contextos, los sujetos podrían considerar que algunas contradicciones pueden ser ciertas. Por ejemplo, en un experimento reciente, Alxatib y Pelletier (2011) mostraron que para casos límite respecto a predicados vagos, muchas personas están dispuestas a afirmar que alguien es "alto y no alto". De forma análoga, otros afirman que no es ni alto ni no-alto. Este tipo de intuiciones preteóricas sobre

Revista de Humanidades de Valparaiso, 2020, No 16, 239-253

(c) (i) $(9)-\mathrm{O}$ CC BY-NC-ND 
conceptos ordinarios pueden servir como base para teorías, como sucede en el caso de Cobreros et al. (2012) que lo toman a favor de su teoría ST. Claro está que las intuiciones preteóricas no alcanzan para generar una teoría lógica entera, pero pueden servir como material de inspiración o de contrastación.

Por otro lado, las lógicas no-clásicas no siempre nacen de intuiciones preteóricas. Las intuiciones teóricas de los expertos también pueden servir como base. Dummett (1978), por ejemplo, considera que oraciones sobre un futuro o pasado lejano no son ni verdaderas ni falsas. Esto funciona como razón a favor del intuicionismo. Filósofos como Williamson no comparten esta intuición teórica, y por eso se inclinan más a favor de preservar el principio del tercero excluido.

\section{2. ¿Intuiciones preteóricas sobre verdad y no sobre validez?}

Ciertamente, tenemos intuiciones preteóricas útiles sobre el concepto de Verdad. También tenemos intuiciones preteóricas sobre los conectivos lógicos; por ende, tenemos intuiciones confiables sobre la preservación esquemática de la verdad. ¿Por qué, entonces, no podemos decir que tenemos intuiciones preteóricas sobre la validez?

Esta objeción involucra una falacia. Tener intuiciones preteóricas sobre los componentes de la definición de validez no implica tener intuiciones preteóricas que nos indiquen cómo definir la validez. Ciertamente, el hecho de que definimos Validez como preservación de verdad nos autoriza a usar nuestras intuiciones preteóricas para evaluar muchos argumentos. Y esto es realmente útil en nuestra disciplina. Por ejemplo, McGee (1985) apela a nuestras intuiciones para probar que, en un caso particular, las premisas de un Modus Ponens son verdaderas, y la conclusión es falsa. Pero puede mostrarse que esa noción de validez como preservación de verdad no está en juego aquí; las intuiciones que necesita McGee son sobre oraciones comunes, sin vocabulario teórico. Del mismo modo, un hedonista, luego de definir lo bueno como aquello que nos da placer, puede apelar a las intuiciones sobre qué cosas hipotéticamente nos causan sufrimiento o placer, para ayudarnos a sistematizar qué cosas son buenas o malas. Pero no apeló a las intuiciones para realizar la definición de "bueno".

\subsection{Conectivos como reglas de introducción y eliminación}

La próxima objeción que voy a considerar tiene un espíritu inferencialista. Si creemos que el significado de los conectivos consiste en sus reglas de introducción y eliminación, entonces si podemos usar nuestras intuiciones preteóricas para determinar el significado de ellas, también podemos usarlas para configurar la teoría lógica completa. Esto se debe a que el sistema podría consistir en meras reglas de introducción y eliminación (esto 
sucede, por ejemplo, en lógica minimal). Esa clase de estrategias fueron adoptadas por Peacocke (2000) y Boghossian (2000) para probar que tenemos un conocimiento a priori de las reglas lógicas, a partir de nuestro uso de las expresiones lógicas.

A esto puedo responder, en primer lugar, algo similar a lo que decía antes. No debe sorprendernos que los hablantes que tienen el concepto de conjunción saben, por ejemplo, que "llueve y hace frío" es verdadero si y sólo si "llueve" y "hace frío" son verdaderos. Pero en la evaluación preteórica del razonamiento, no aparecen solamente estas consideraciones preteóricas, sino también otras: como efecto de esto, muchos hablantes competentes son incompetentes para evaluar piezas de razonamiento lógico. Es el teórico quien decide basar la validez en las condiciones de verdad de los conectivos, aquí expresadas por las reglas de introducción y eliminación. Es también el teórico quien decide liberar el concepto de los factores contextuales o pragmáticos, por ejemplo.

En segundo lugar, las reglas adicionales como Doble Negación o ex falso sequitur quodlibet prueban que la lógica no puede reducirse a las reglas que supuestamente constituyen el significado de los conectivos. Como bien sabemos, las reglas de introducción y eliminación tradicionales de los conectivos clásicos solo nos permiten obtener lógica minimal. En particular, no hay ninguna regla que nos permita inferir cualquier cosa a partir de una contradicción. Sin embargo, aceptamos esa regla siempre que tengamos el concepto teórico de validez y nos demos cuenta que la inferencia ex falso sequitur quodlibet preserva verdad.

\subsection{Revisión del concepto de validez}

La última objeción a mi propuesta es que algunas lógicas no aceptan la noción de validez como preservación de verdad. Por ejemplo, algunos relevantistas consideran que para que un argumento sea válido, las premisas deben ser informativamente relevantes para la conclusión. Algunos intuicionistas adoptan un concepto de validez donde debe preservarse la demostrabilidad, no la verdad.

Esto podría motivar dos objeciones a mi propuesta: primero, si nuestras intuiciones preteóricas sobre la validez son tomadas como razones para rechazar la noción clásica de validez, entonces (Extremo) estaría en lo correcto al final. En segundo lugar, si el concepto de validez no menciona a la verdad, nuestras intuiciones sobre el significado de los conectivos (que presuponen una noción veritativo-condicional del significado) podrían ser de poca ayuda para la lógica.

No creo que debamos preocuparnos demasiado sobre el primer problema. Pues, si caracterizamos la validez sin usar el concepto de preservación de verdad, entonces la justificación no puede ser preteórica, porque (como argumenté) carecemos de un concepto preteórico uniforme y claro de validez. Podemos ver qué sucede con la lógica relevante, como ejemplo ilustrativo. Esta lógica fue propuesta como una noción más intuitiva de la 
Intuiciones en lógica: una propuesta moderada

Diego Tajer

consecuencia lógica, pero al final, nuestras supuestas intuiciones preteóricas sobre la validez no concuerdan demasiado con la consecuencia relevantista: podemos intuitivamente rechazar ex falso, pero cuando tenemos que rechazar el silogismo disyuntivo nuestras intuiciones se vuelven más bien oscuras ${ }^{6}$. También queda claro aquí que las intuiciones son juicios o disposiciones a juzgar, y por eso pueden fallar; justamente la teoría puede mostrar cómo ciertas intuiciones preteóricas llevan al fracaso o la incoherencia. Si las intuiciones fueran naturalmente confiables y conectadas con la verdad (como sugieren los racionalistas), el hecho de que relevantistas y clásicos tengan intuiciones distintas sería incoherente. Como Mares (2004) y otros lógicos relevantistas argumentaron, para entender la posición relevantista hace falta explicitar y justificar la interpretación filosófica de los modelos que tenemos en mente ${ }^{7}$; no basta con mostrar que algunos argumentos clásicos son anti-intuitivos, como intentaron hacer los relevantistas originalmente. Otro caso interesante es el de la lógica intuicionista; a diferencia de los relevantistas, los intuicionistas no suelen presentar su teoría como una reconstrucción adecuada de los conceptos del lenguaje natural; por el contrario, sus desarrollos parten siempre de complejas consideraciones filosóficas sobre la naturaleza del conocimiento y de la realidad (Brouwer 1948), que no son necesariamente "intuitivas" a primera vista.

El segundo problema también puede responderse. Incluso si cambiamos el concepto de validez por otro, las intuiciones preteóricas sobre los conectivos podrían seguir jugando un rol. Si (por ejemplo) adoptamos una noción relevantista de validez usando semántica de situaciones, entonces nuestras intuiciones preteóricas sobre la "adecuación en situación" de las aserciones va a jugar un rol importante. Por otro lado, incluso si adoptamos una noción intuicionista de validez, donde se preserva la verificabilidad en vez de la verdad, todavía podríamos defender la utilidad de las intuiciones preteóricas para determinar el significado de los conectivos, porque la condición de verificación de una oración como $\neg \mathrm{p}$ o $\mathrm{p} \wedge \mathrm{q}$ puede ser considerada (para el intuicionista) también preteórica ${ }^{8}$.

\section{Conclusión}

He argumentado a favor de una posición moderada sobre el rol de las intuiciones en lógica. De acuerdo con esta posición, la lógica no pretende respetar las intuiciones preteóricas sobre la validez. La noción ordinaria de validez es un concepto ramificado, que mezcla asuntos semánticos, pragmáticos y contextuales. Por ende, no podemos esperar que un análisis conceptual de nuestras intuiciones sobre la validez nos arroje un concepto teórico uniforme. Por el contrario, los conectivos lógicos son (en general) usados de manera más

\footnotetext{
${ }^{6}$ Véase Woods (2002) para una discusión sobre este punto.

${ }^{7}$ Este debate está abierto pero excede el alcance de este artículo.

${ }^{8}$ De hecho, podemos considerar al intuicionismo como una lógica que no revisa la noción de consecuencia sino que establece condiciones de verdad más fuertes para las oraciones.
}

Revista de Humanidades de Valparaíso, 2020, No 16, 239-253

(c) $(i) \Theta(9)$ CC BY-NC-ND 
homogénea por los hablantes competentes. Por lo tanto, las intuiciones preteóricas sobre éstos pueden ser útiles para revisar la estipulación de su significado en distintas teorías lógicas, y es metodológicamente correcto usarlas como evidencia.

\section{Referencias bibliográficas}

Alxatib, Sam \& Pelletier, Francis (2011). The psychology of vagueness: borderline cases and contradictions. Mind and Language, 26(3), 287-326. https://doi.org/10.1111/j.14680017.2011.01419.x

Bealer, G. (1998). Intuition and the autonomy of philosophy. En M. DePaul y W. Ramsay (eds.), Rethinking intuition, pp. 201-239. Maryland: Rowman and Littlefield. URL: https://www. amazon.com/-/es/Michael-DePaul/dp/0847687961

Beall, JC y Restall, G. (2006). Logical Pluralism Oxford: Oxford University Press. https://doi. org/10.1093/acprof:oso/9780199288403.001.0001

Boghossian, P. (2000). Knowledge of logic. En P. Boghossian y C. Peacocke, (eds.), New Essays on the A Priori, pp. 229-254. Oxford: Oxford Clarendon Press. https://doi. org/10.1093/0199241279.001.0001

Brouwer, L.E.J. (1948). Consciousness, philosophy, and mathematics. International Philosophy Congress 1988 (Amsterdam), 1235-1249. En Brouwer, L.E.J. 1975, Collected Works, Vol. 1. Ed. A. Heyting, North Holland, Amsterdam: pp. 480-494. URL: https://www.amazon. com/Collected-Works-Philosophy-Foundations-Mathematics/dp/0720420768

Cappelen, H. (2012). Philosophy without intuitions. Oxford: Oxford University Press. URL: https://www.amazon.com/-/es/Herman-Cappelen/dp/0199644861

Chudnoff, E. (2011). The Nature of Intuitive Justification. Philosophical Studies, 163, 313-333. https://doi.org/10.1007/s11098-010-9495-2

Cobreros, P., Egré, P, Ripley, D. \& van Rooij, R. (2012). Tolerant, classical, strict. Journal of Philosophical Logic, 41, 347-385. https://doi.org/10.1007/s10992-010-9165-z

Cummins, R. (1998). Reflections of Reflective Equilibrium. En M. DePaul y W. Ramsay (eds.), Rethinking intuition, pp. 113-127. Maryland: Rowman and Littlefield. URL: https://www. amazon.com/-/es/Michael-DePaul/dp/0847687961

Dutilh Novaes, C. (2011) The myth of the pre-theoretical notion of logical consequence. Charla en la Universidad de St. Andrews, April 5 2011. URL: https://www.newappsblog. com/2011/03/the-myth-of-the-pre-theoretical-notion-of-logical-consequence.html

Dummett, M. (1978). Truth and other enigmas. Cambridge MA: Harvard University Press. URL: https://www.hup.harvard.edu/catalog.php?isbn=9780674910768 
Intuiciones en lógica: una propuesta moderada

Diego Tajer

Evans, J. S. B. T. (2005). Deductive Reasoning. En K. J. Holyoak \& R. G. Morrison (Eds.), The Cambridge handbook of thinking and reasoning, pp. 169-184. Cambridge University Press. URL: https://www.amazon.de/Cambridge-Handbook-Reasoning-Handbooks-Psychology/dp/0521531012

Field, Hartry (2009). Saving truth from paradox. Oxford: Oxford University Press. https://doi. org/10.1093/acprof:oso/9780199230747.001.0001

Goodman, Nelson (1965). Fact, Fiction and Forecast. Cambridge MA: Harvard University Press. https://doi.org/10.2307/2964684

Horsten, Leon (2011). The Tarskian Turn: Deflationism and Axiomatic Truth. Cambridge MA: MIT Press. https://doi.org/10.7551/mitpress/9780262015868.001.0001

Jané, I. (2005). What is Tarski's common concept of logical consequence? The Bulletin of Symbolic Logic, 12, 1-42. https://doi.org/10.2178/bsl/1140640942

Kripke, S. (1980). Naming and Necessity. Cambridge MA: Harvard University Press. https://doi. org/10.1017/S0031819100050427

Kripke, S. (1975). Outline of a Theory of Truth. Journal of Philosophy, 72, 690-716. https://doi. org/10.2307/2024634

Mares, E. (2004), Relevant Logic, Cambridge, Cambridge University Press. https://doi. org/10.1017/CBO9780511520006

Martin, B. (2020). Identifying logical evidence. Synthese, en prensa. https://doi.org/10.1007/ s11229-020-02618-y

McGee, V. (1985). A counter-example to modus ponens. Journal of Philosophy, 82, 462-471. https://doi.org/10.2307/2026276

Mercier, H. y Sperber, D. (2011). Why do humans reason? Behavioral and Brain Sciences, 34, 57-111. https://doi.org/10.1017/S0140525X10000968

Peacocke, C. (2000). Explaining the A Priori: the programme of moderate rationalism. En Boghossian, P. y Peacocke, C. (eds.), New Essays on the A Priori, pp. 255-285. Oxford: Oxford Clarendon Press. doi: 10.1093/0199241279.001.0001

Priest, Graham (2006). Doubt Truth to be a Liar. Oxford: Oxford University Press. https://doi. org/10.1093/0199263280.001.0001

Sosa, E. (1998). Minimal intuition. En M. DePaul y W. Ramsay (eds.), Rethinking intuition, pp. 257-269. Maryland: Rowman and Littlefield. URL: https://www.amazon.com/-/es/Michael-DePaul/dp/0847687961

Tarski, A. (1936). On the concept of following logically (traducido al inglés por M. Stroinska and D. Hitchcock). History and Philosophy of Logic, 23, 155-196, 2002. https://doi. org/10.1080/0144534021000036683

Weatherson, B. (2003). What good are counterexamples? Philosophical Studies, 140, 1-31. https://doi.org/10.1023/A:1024961917413

Revista de Humanidades de Valparaíso, 2020, No 16, 239-253

(c) (1) @९ $\mathrm{CC}$ BY-NC-ND 


$$
\begin{gathered}
\text { Intuiciones en lógica: una propuesta moderada } \\
\text { Diego Tajer }
\end{gathered}
$$

Weinberg, J.M., Nichols, S. y Stich, S. (2001). Normativity and Epistemic Intuitions. Philosophical Topics, 29, 429-460. https://doi.org/10.5840/philtopics2001291/217

Williamson, Timothy (2007). The philosophy of philosophy. New Jersey: Blackwell Publishing. https://doi.org/10.1002/9780470696675

Woods, J. (2002). Paradox and Paraconsistency: Conflict Resolution in the Abstract Sciences. Cambridge: Cambridge University Press. https://doi.org/10.1017/CBO9780511614002 
